\title{
ARTICLES
}

\section{Absolute-energy-minimum principles for linear-scaling electronic-structure calculations}

\author{
Weitao Yang* \\ Department of Chemistry, The Hong Kong University of Science and Technology, Clear Water Bay, Kowloon, Hong Kong, China
}

(Received 1 May 1997)

\begin{abstract}
Two absolute energy minimum principles are developed for first-principle linear-scaling electronic structure calculations. One is with a normalization constraint and the other without any constraint. The density matrix is represented by a set of nonorthogonal localized orbitals and an auxiliary matrix which at the minimum becomes a generalized inverse of the overlap matrix of the localized orbitals. The number of localized orbitals is allowed to exceed the number of occupied orbitals. Comparison with other variational principles is made and numerical tests presented. [S0163-1829(97)01939-5]
\end{abstract}

Most electronic structure calculations are based on a oneelectron Hamiltonian, either within semiempirical approaches such as tight-binding and semiempirical quantum chemical models, or within first-principle approaches such as the density functional and Hartree-Fock-based method. The one-electron Hamiltonian provides building blocks for the construction of the electronic wave function or the electron density. Conventional direct and iterative diagonalization algorithms for obtaining eigenvalues require an overall computational effort which grows as the cubic of the number of atoms. This has been a major limiting factor for the application of the electronic structure methods to large systems.

The possibility of obtaining the sum of eigenvalues in electronic structure calculations with effort scaling linearly with the size of the system was first demonstrated in the divide-and-conquer approach. ${ }^{1,2}$ Subsequently, there has been a surge of interest in linear scaling methods for this problem. ${ }^{3-13}$ The key to achieving linear scaling is the localization of the electronic degrees of freedom; namely, it is necessary to limit to a local region of space the physical span of electronic degrees of freedom. Methods for the localization developed so far can be grouped into two categories. The local Hamiltonian approaches obtain the local properties, such as the electron density and the kinetic energy density, from the one-electron potential locally, bypassing solution to the eigenequations. ${ }^{1,2,10-12}$ The variational approaches are based on the reformulation of the conventional constrained variational principles and the truncation of the density matrix or the localized orbitals. ${ }^{3-9}$ The reformulated variational principles usually are only local minimum principles, ${ }^{4,5}$ with the exception of Kohn's global minimum principle for noninteracting electrons. ${ }^{8}$ There is a need for absolute minimum principles, particularly for interacting electrons.

In this paper, we propose two absolute minimum principles for linear scaling electronic structure calculations with both tight-binding and first-principle approaches. One is with a normalization constraint and the other without any constraint. Consider a system with $N$ electrons in a spincompensated situation (the extension to spin-polarized case is trivial). For most electronic structure calculations including density functional, Hartree-Fock, and semiempirical methods, the ground state energy $E(N)$ can be expressed in terms of $E[\hat{\rho}]$, a functional of the one-electron density operator $\hat{\rho}$. For example, $E[\hat{\rho}]=2 \operatorname{Tr}(\hat{h} \hat{\rho})$, for noninteracting electrons with a Hamiltonian $\hat{h}$. The energy minimum principle is

$$
E(N)=\min E[\hat{\rho}]
$$

where $\hat{\rho}$ has to satisfy the following conditions:

$$
\begin{gathered}
\text { Hermitian: } \hat{\rho}=\hat{\rho}^{\dagger}, \\
\text { idempotency: } \hat{\rho}^{2}=\hat{\rho}, \\
\text { normalization: } 2 \operatorname{Tr}(\hat{\rho})=N .
\end{gathered}
$$

Given a set of N/2 linearly independent orbitals $\{\psi\}$, which are not necessarily orthonormal, then one can construct a density operator which obeys all the above three conditions, namely,

$$
\hat{\rho}_{N / 2}=\sum_{i, j}^{N / 2}\left|\psi_{i}\right\rangle\left(S^{-1}\right)_{i j}\left\langle\psi_{j}\right|,
$$

where $\mathbf{S}^{-1}$ is the inverse of the overlap matrix $\mathbf{S}, S_{i j}=\left\langle\psi_{i} \mid \psi_{j}\right\rangle$. The minimization over $\hat{\rho}_{N / 2}$ in Eq. (1) can then be accomplished by minimization over the orbitals $\{\psi\}$, without any constraint. This construction is well known. However, it does not directly lead to a linear scaling algorithm, because of the $\mathbf{S}^{-1}$ matrix involved.

A variational principle used in conjunction with the spatial truncation of localized orbitals leads to a linear scaling algorithm. The desired variational principle should not use $\mathbf{S}^{-1}$ and should allow the number of localized orbitals to exceed $N / 2$, the number of occupied orbitals. The latter is important for eliminating the multiminimum problem associated with the truncation of the localized orbitals. ${ }^{4}$ We now generalize Eq. (5) and build a density operator from a set of 
arbitrary $M$ (possibly linearly dependent) orbitals $\left\{\psi_{i}, i=1, \ldots, M\right\}$, where $M \geqslant N / 2$. Consider

$$
\hat{\rho}=\sum_{i, j}^{M}\left|\psi_{i}\right\rangle\left(S^{-}\right)_{i j}\left\langle\psi_{j}\right|,
$$

where $\mathbf{S}^{-}$is the (1) inverse of the overlap matrix $\mathbf{S}$, defined by the following matrix equation:

$$
\mathbf{S S}^{-} \mathbf{S}=\mathbf{S}
$$

The (1) inverse is the simplest one of the several generalized inverses for a singular matrix. ${ }^{14}$ Note that $\mathbf{S}$ is singular when the orbitals $\{\psi\}$ are linearly dependent.

We shall show that $\hat{\rho}$ defined in Eq. (6) is idempotent and Hermitian. Take arbitrary orbitals $\psi_{k}$ and $\psi_{l}$ belonging to the set of orbitals $\{\psi\}$, and another arbitrary orbital $\psi_{\perp}$ orthogonal to $\{\psi\}$, that is, $\left\langle\psi_{\perp} \mid \psi_{i}\right\rangle=0$, for $i=1, \ldots, M$. Then

$$
\hat{\rho}\left|\psi_{\perp}\right\rangle=0
$$

and

$$
\left\langle\psi_{k}|\hat{\rho}| \psi_{l}\right\rangle=\sum_{i, j}^{M}\left\langle\psi_{k} \mid \psi_{i}\right\rangle\left(S^{-}\right)_{i j}\left\langle\psi_{j} \mid \psi_{l}\right\rangle=\left\langle\psi_{k} \mid \psi_{l}\right\rangle
$$

where we use the definition of the (1) inverse, Eq. (7). The above two equations imply that

$$
\hat{\rho}\left|\psi_{k}\right\rangle=\left|\psi_{k}\right\rangle .
$$

It follows that $\hat{\rho}^{2}\left|\psi_{k}\right\rangle=\hat{\rho}\left|\psi_{k}\right\rangle$, and $\hat{\rho}^{2}\left|\psi_{\perp}\right\rangle=\hat{\rho}\left|\psi_{\perp}\right\rangle=0$. Therefore, the idempotency condition Eq. (3) is satisfied by the density operator defined in Eq. (6). For the Hermitian condition, it suffices to show that $\hat{\rho}^{\dagger} \hat{\rho}=\hat{\rho}^{\dagger}$, which follows from Eqs. (8) and (9).

The only remaining constraint for the density operator is the normalization condition

$$
2 \operatorname{Tr}(\hat{\rho})=2 \operatorname{Tr}\left(\mathbf{S}^{-} \mathbf{S}\right)=N
$$

or

$$
2 \operatorname{rank}(\mathbf{S})=N \text {, }
$$

where $\operatorname{rank}(\mathbf{S})$ is the rank of the $\mathbf{S}$ matrix. The rank of the $\mathbf{S}$ matrix is just the number of linearly independent column or row vectors in the $\mathbf{S}$ matrix. Since $S_{i j}=\left\langle\psi_{i} \mid \psi_{j}\right\rangle, \operatorname{rank}(\mathbf{S})$ is also equal to the number of linearly independent orbitals in the set $\left\{\psi_{i}, i=1, \ldots, M\right\}$. In Eq. (10), we use the equality $\operatorname{rank}(\mathbf{S})=\operatorname{Tr}\left(\mathbf{S}^{-} \mathbf{S}\right){ }^{14}$ The construction of an idempotent and Hermitian density operator through Eq. (6) appears to be a new result.

We define a functional of $M$ orbitals $\left\{\psi_{i}, i=1, \ldots, M\right\}$ and of a $M \times M$ matrix $\mathbf{A}$ :

$$
\Omega[\mathbf{A},\{\psi\}]=E\left[\hat{\rho}_{\mathbf{A}}\right]-2 \eta \operatorname{Tr}\left(\hat{\rho}_{\mathbf{A}}\right)
$$

where

$$
\hat{\rho}_{\mathbf{A}}=\sum_{i, j}^{M}\left|\psi_{i}\right\rangle A_{i j}\left\langle\psi_{j}\right|
$$

and $\eta$ is a constant to be specified. The role of the constant $\eta$ in constructing the desired variational principles will be shown later. If $\mathbf{A}=\mathbf{S}^{-}, \hat{\rho}_{\mathbf{A}}$ given by Eq. (6) is Hermitian and idempotent, and the $N$-electron ground state energy can then be written as

$$
E(N)=\min _{\{\psi\}, 2 \operatorname{rank}(\mathbf{S})=N} \Omega\left[\mathbf{S}^{-},\{\psi\}\right]+\eta N
$$

where the normalization condition remains the only constraint.

Now we use a trick of Mauri et al. ${ }^{3}$ and introduce $\mathbf{A}(\lambda)=\lambda\left[(2 \mathbf{X}-\mathbf{S X S})-\mathbf{S}^{-}\right]+\mathbf{S}^{-}$. Then

$$
\begin{aligned}
\Delta \Omega & =\Omega[(2 \mathbf{X}-\mathbf{S X S}),\{\psi\}]-\Omega\left[\mathbf{S}^{-},\{\psi\}\right] \\
& =\int_{0}^{1} d \lambda \partial \Omega[\mathbf{A}(\lambda),\{\psi\}] / \partial \lambda \\
& =\int_{0}^{1} d \lambda \sum_{i, j}^{M} \frac{\partial \Omega[\mathbf{A}(\lambda),\{\psi\}]}{\partial A_{i j}(\lambda)} \frac{\partial A_{i j}(\lambda)}{\partial \lambda} \\
& =2 \operatorname{Tr}\left\{(\overline{\mathbf{H}}-\eta \mathbf{S})\left[(2 \mathbf{X}-\mathbf{S X S})-\mathbf{S}^{-}\right]\right\},
\end{aligned}
$$

where $\overline{\mathbf{H}}$ is the $\lambda$-averaged Hamiltonian matrix, $\bar{H}_{j i}=\frac{1}{2} \int_{0}^{1} d \lambda \partial E\left[\hat{\rho}_{\mathbf{A}(\lambda)}\right] / \partial A_{i j}(\lambda)$, where $\hat{\rho}_{\mathbf{A}(\lambda)}$ is defined by Eq. (12). It is also possible to express $\bar{H}_{j i}=\left\langle\psi_{j}|\hat{h}| \psi_{i}\right\rangle$, with $\hat{h}=-\nabla^{2} / 2+\bar{v}$, and $\bar{v}=\int_{0}^{1} d \lambda v(\lambda)$, where $v(\lambda)$ is a oneelectron potential defined in the appropriate theory and evaluated with the density matrix $\hat{\rho}_{\mathbf{A}(\lambda)}$. For noninteracting electrons, $\bar{v}$ is just the external potential. For densityfunctional theory, $v(\lambda)$ is the Kohn-Sham potential evaluated with the density matrix $\hat{\rho}_{\mathbf{A}(\lambda)}$.

To obtain the desired variational principles, we need the following variational expression:

$$
\operatorname{Tr}\left(\mathbf{B S}^{-}\right)=\min _{\mathbf{X}=\mathbf{X}^{\dagger}} \operatorname{Tr}[\mathbf{B}(2 \mathbf{X}-\mathbf{X S X})]
$$

for any negative definite matrix $\mathbf{B}$ that can be expressed by $B_{i j}=\left\langle\psi_{i}|\hat{b}| \psi_{j}\right\rangle$ with some operator $\hat{b}$. The auxiliary $M \times M$ matrix $\mathbf{X}$ is Hermitian. Let $\mathbf{X}=\mathbf{X}_{0}+\Delta$, and make the expansion in $\Delta$ :

$$
\begin{aligned}
\operatorname{Tr}[\mathbf{B}(2 \mathbf{X}-\mathbf{X S X})]= & \operatorname{Tr}\left[\mathbf{B}\left(2 \mathbf{X}_{0}-\mathbf{X}_{0} \mathbf{S} \mathbf{X}_{0}\right)\right] \\
& +\operatorname{Tr}\left[\mathbf{B}\left(2 \boldsymbol{\Delta}-\Delta \mathbf{S} \mathbf{X}_{0}-\mathbf{X}_{0} \mathbf{S} \Delta\right)\right] \\
& -\operatorname{Tr}[\mathbf{B} \boldsymbol{\Delta} \mathbf{S} \boldsymbol{\Delta}]
\end{aligned}
$$

Note that $\mathbf{B S}^{-} \mathbf{S}=\mathbf{B}$, following from Eq. (9). The minimizer is $\mathbf{X}_{0}=\mathbf{S}^{-}$, which makes the linear term in $\boldsymbol{\Delta}$ vanish. The quadratic term, $-\operatorname{Tr}[\mathbf{B} \boldsymbol{\Delta} \mathbf{S} \boldsymbol{\Delta}]$, is non-negative for any Hermitian $\boldsymbol{\Delta}$, because the matrix $\mathbf{S}$ is positive semidefinite and the matrix $\mathbf{B}$ is negative definite. We have thus proved Eq. (15).

Set the constant $\eta$ in Eq. (14) such that the matrix $(\overline{\mathbf{H}}-\eta \mathbf{S})$ is negative definite. This can be achieved by letting the real constant $\eta$ be greater than the largest eigenvalue of the generalized eigenvalue problem formed from the Hamil- 
tonian $\overline{\mathbf{H}}$ and the overlap matrix $\mathbf{S}$. Now that $(\overline{\mathbf{H}}-\eta \mathbf{S})$ is negative definite, we can apply Eq. (15) to Eq. (14). We then have $\Delta \Omega \geqslant 0$, or

$$
\Omega\left[\mathbf{S}^{-},\{\psi\}\right] \leqslant \Omega[(2 \mathbf{X}-\mathbf{X S X}),\{\psi\}],
$$

for any Hermitian $\mathbf{X}$, with the equality satisfied by $\mathbf{X}=\mathbf{S}^{-}$. Thus, from Eqs. (13) and (16), we obtain the first desired absolute energy minimum principle in terms of $\left\{\psi_{i}, i=1, \ldots, M\right\}$ and $\mathbf{X}$, with only the normalization constraint

$$
E(N)=\min _{\{\psi\}, 2 \operatorname{rank}(\mathbf{S})=N \mathbf{X}=\mathbf{X}^{\dagger}} \min \Omega[(2 \mathbf{X}-\mathbf{X S X}),\{\psi\}]+\eta N .
$$

Compared with the variational principle of Eq. (13), here we use an expression (2X-XSX) instead of $\mathbf{S}^{-}$, the (1) inverse of the overlap matrix $\mathbf{S}$. Because $\mathbf{S}^{-}$is difficult to evaluate, we cannot directly apply the variational principle of Eq. (13). The Hermitian matrix $\mathbf{X}$ is used here as an auxiliary matrix which at the minimum becomes $\mathbf{S}^{-}$.

We would like to eliminate the normalization constraint in Eq. (17). For $N$ noninteracting electrons, the ground state energy $E(N)$ is just the sum of the lowest eigenvalues, $E(N)=2 \Sigma_{i}^{N / 2} \epsilon_{i}$. We can choose a chemical potential $\mu$ such that $\epsilon_{N / 2}<\mu<\epsilon_{N / 2+1}$. Then for an arbitrary $N^{\prime}$, $2 \Sigma_{i}^{N / 2}\left(\epsilon_{i}-\mu\right) \leqslant 2 \sum_{i}^{N^{\prime} / 2}\left(\epsilon_{i}-\mu\right)$, which can be written as

$$
E(N)-\mu N \leqslant E\left(N^{\prime}\right)-\mu N^{\prime} .
$$

Equation (18) derived here for noninteracting electrons is also in general taken to be valid for atom and molecules and is the basis for density-functional theory of the zerotemperature grand canonical ensemble. ${ }^{15,16}$ If we assume the same, it allows us to release the normalization constraint in varying the orbitals $\{\psi\}$. Combining the Eqs. (17) and (18), we obtain the second desired absolute energy minimum principle in terms of $\left\{\psi_{i}, i=1, \ldots, M\right\}$ and $\mathbf{X}$, without any constraint:

$$
\begin{aligned}
E(N)= & \min \{\min \Omega[(2 \mathbf{X}-\mathbf{X S X}),\{\psi\}]+2(\eta-\mu) \operatorname{rank}(\mathbf{S})\} \\
& \{\psi\} \quad \mathbf{X}=\mathbf{X}^{\dagger} \\
& +\mu N .
\end{aligned}
$$

The variational principles of Eqs. (17) and (19) are our main results. We now discuss their properties.

(1) Our variational principles define the ground state energy as the absolute minimum, which can provide more robust minimization algorithms than local minimum principles. The number of localized orbitals here is permitted to exceed the number of occupied orbitals, a property deemed critical to eliminate the multi-minimum problem associated with the truncation of the localized orbitals in the physical space. ${ }^{4}$ The minimizing orbitals are also allowed to be nonorthogonal, which facilitates optimal localization. ${ }^{7}$

(2) Both variational principles contain $\operatorname{rank}(\mathbf{S})$. To carry out the minimization, we need an explicit formula. We have found it convenient to use a variational expression

$$
\operatorname{rank}(\mathbf{S})=\operatorname{Tr}\left(\mathbf{S S}^{-}\right)=-\underset{\mathbf{X}=\mathbf{X}^{\dagger}}{\min } \operatorname{Tr}[(-\mathbf{S})(2 \mathbf{X}-\mathbf{X S X})],
$$

which follows from Eq. (15). In this way, we only need to carry out one minimization with $\mathbf{X}$, either for the energy as in Eq. (17) and in Eq. (19) or for $\operatorname{rank}(\mathbf{S})$ as in Eq. (20). Both minimization procedures over $\mathbf{X}$ are based on the same variational principle, Eq. (15): for the energy, $\mathbf{B}=(\overline{\mathbf{H}}-\eta \mathbf{S})$, and for $\operatorname{rank}(\mathbf{S}), \mathbf{B}=-\mathbf{S}$. In both cases, the minimizing $\mathbf{X}$ matrices are the same and identical to $\mathbf{S}^{-}$.

(3) In the special case of $M=N / 2$, the orbitals $\left\{\psi_{i}, i=1, \ldots, N / 2\right\}$ are linearly independent. Then the normalization constraint is satisfied, and Eq. (17) becomes

$$
E(N) \leqslant \Omega[(2 \mathbf{X}-\mathbf{X S X}),\{\psi\}]+\eta N,
$$

for $\left\{\psi_{i}, i=1, \ldots, N / 2\right\}$ and any $\mathbf{X}=\mathbf{X}^{\dagger}$. Furthermore, the equality can be realized by varying $\{\psi\}$ for a fixed $\mathbf{X}$; this follows from that the density matrix is invariant with respect to an orthogonal transformation of the orbitals and fixing $\mathbf{X}$ makes the energy minimizing orbitals to satisfy $\mathbf{S}^{-1}=\mathbf{X}$. In particular, when $\mathbf{X}$ is an identity matrix, we obtain the variational principle of Mauri et al. and Ordejon et al. ${ }^{3,6}$ Here, Eq. (21) allows the minimizing orbitals to be nonorthogonal. Hierse et al. proposed using non-orthogonal orbitals and a variational principle similar to Eq. (21). ${ }^{7}$ But in their derivation of the variational principle, $\mathbf{X}$ is required to be positive definite. In Eq. (21), $\mathbf{X}$ only needs to be Hermitian.

(4) If we use Eqs. (11) and (20) in Eq. (19) and ignore the three minimization procedures, the right-hand side of Eq. (19) is an energy functional

$$
W(\mathbf{X},\{\psi\}, M)=E\left[\hat{\rho}_{\mathbf{A}}\right]-2 \mu \operatorname{Tr}\left(\hat{\rho}_{\mathbf{A}}\right)+\mu N,
$$

where $\hat{\rho}_{\mathbf{A}}$ is given by Eq. (12) with $\mathbf{A}=2 \mathbf{X}-\mathbf{X S X}$. For noninteracting electrons with a Hamiltonian $\hat{h}$, Eq. (22) becomes

$$
W_{0}(\mathbf{X},\{\psi\}, M)=2 \operatorname{Tr}[(\mathbf{H}-\mu \mathbf{S})(2 \mathbf{X}-\mathbf{X S X})]+\mu N,
$$

where $H_{i j}=\left\langle\psi_{i}|\hat{h}| \psi_{j}\right\rangle$. With the appropriate value for $\mu$ and at the ground state energy of $N$ electrons, $W_{0}$ has a local minimum for variation in arbitrary $\{\psi\}$ and in a Hermitian $\mathbf{X}$; we can show this by expanding $W_{0}$ to the second order in $\mathbf{X}$ and $\{\psi\}$, but we omit the details here. Note that when $\mathbf{X}$ is an identity matrix, Eq. (23) becomes the local-minimum variational expression of Kim et al. for noninteracting electrons. ${ }^{4}$ Equation (23) here allows the minimizing orbitals to be nonorthogonal, because $\mathbf{X}$ can be different from an identity matrix.

(5) We would like to address the role of the chemical potential $\mu$ in our variational principle, Eq. (19). Different values of $\mu$ used in the minimization lead to energies corresponding to different numbers of electrons. Therefore, in a practical implementation, one needs to adjust the value of $\mu$ in order to obtain the desired number of electrons, as in other variational principles involving with a chemical potential. ${ }^{4,5,8}$

For numerical tests of Eq. (19), we consider a tetraglycine molecule of 31 of atoms and 96 electrons $(N=96)$, within the semiempirical quantum chemical method (PM3) of the MOPAC program. ${ }^{13}$ For simplicity, we only take as input the Hamiltonian $\hat{h}$ resulting from a self-consistent calculation so that energy functional is now in a noninteracting electron form, $E[\hat{\rho}]=2 \operatorname{Tr}(\hat{h} \hat{\rho})$. The variational principle of Eq. (19) 


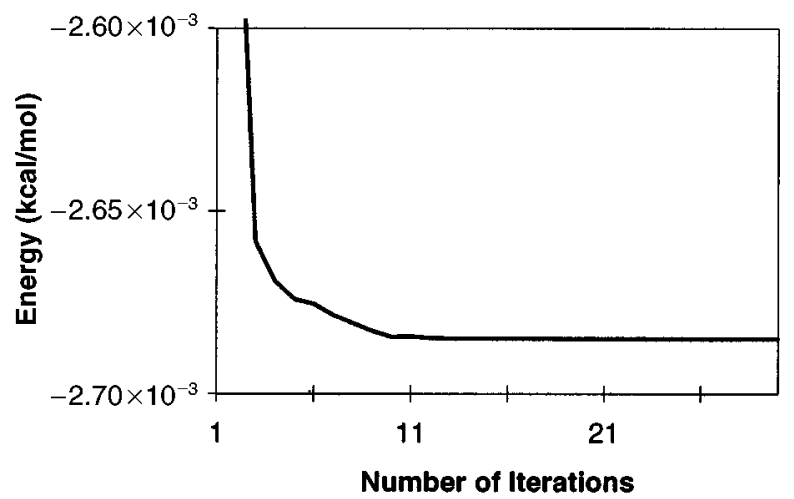

FIG. 1. The total energy as a function of the number of iterations for the tetraglycine molecule.

is used in conjunction with Eq. (20) and with the appropriate values for $\mu$ and $\eta$. The orbitals $\{\psi\}$ are now represented by C, a $N_{\text {ao }} \times M$ coefficient matrix for representing $\{\psi\}$ in terms of the $N_{\text {ao }}$ atomic basis functions. ( $N_{\mathrm{ao}}=82$ for this molecule.) We do not make any truncation for both $\mathbf{C}$ and $\mathbf{X}$, as we would in an $O(N)$ calculation. The conjugate gradient minimization is used throughout. As shown in Eq. (19), the minimization over $\mathbf{C}$ is accompanied by the inner minimization over $\mathbf{X}$; that is, the minimization over $\mathbf{X}$ is carried out within each iteration for $\mathbf{C}$. The minimization over $\mathbf{X}$ can be carried out either for energy as in Eq. (19) or for $\operatorname{rank}(\mathbf{S})$ as in Eq. (20). The procedure is as follows: (i) Random guess for initial $\mathbf{C}$, and identity matrix for initial $\mathbf{X}$; (ii) minimiza- tion over $\mathbf{X}$; and (iii) minimization over $\mathbf{C}$ with inner minimization over $\mathbf{X}$ until convergence.

Figure 1 reports the total energy as a function of the number of conjugate gradient minimization steps in $\mathbf{C}$. The data were obtained in the following calculations: We take $M=20+N / 2$. The minimization over $\mathbf{X}$ has been carried out for $\operatorname{rank}(\mathbf{S})$ as in Eq. (20). For each inner minimization over $\mathbf{X}$ with a given $\mathbf{C}$, we let $n_{\mathbf{X}}=5$, where $n_{\mathbf{X}}$ is the maximum number of iteration. In the initial iterations in $\mathbf{C}$, the number of minimization over $\mathbf{X}$ reached the maximum $n_{\mathbf{X}}$, but it falls to 1 quickly at the 11th iteration in $\mathbf{C}$ because the convergence is reached within the tolerance of $1.0 \times 10^{-6}$ in $\operatorname{rank}(\mathbf{S})$. This indicates the minimization over $\mathbf{X}$ carried out within each iteration for $\mathbf{C}$ is very efficient. The energy is converged to $1.0 \times 10^{-3} \mathrm{~K} \mathrm{cal} / \mathrm{mol}$ at the 28 th iteration. Similar results were obtained when we used other values of $M$ and $n_{\mathbf{X}}$, and when we carried out the minimization over $\mathbf{X}$ for energy as in Eq. (19).

The initial tests here demonstrated that one of the proposed variational principles has given correct results for the test problem. In conjunction with the truncation of both the localized orbitals and the $\mathbf{X}$ matrix, we can thus obtain the corresponding linear scaling algorithms.

Financial support from the National Science Foundation, the NIH Research Resources at the University of North Carolina-Chapel Hill, and the Sloan Foundation is gratefully acknowledged. The author thanks Tai-Sung Lee for providing the Hamiltonian matrices used and Professor YiJing Yan for helpful comments.
*On leave from the Department of Chemistry, Duke University, Durham, North Carolina 27708-0346.

${ }^{1}$ W. Yang, Phys. Rev. Lett. 66, 1438 (1991).

${ }^{2}$ W. Yang and T. Lee, J. Chem. Phys. 163, 5674 (1995).

${ }^{3}$ F. Mauri, G. Galli, and R. Car, Phys. Rev. B 47, 9973 (1993).

${ }^{4}$ J. Kim, F. Mauri, and G. Galli, Phys. Rev. B 52, 1640 (1995).

${ }^{5}$ X.-P. Li, R. W. Nunes, and D. Vanderbilt, Phys. Rev. B 47, 10 891 (1993).

${ }^{6}$ P. Ordejón, D. Drabold, M. Grumbach, and R. M. Martin, Phys. Rev. B 48, 14646 (1993).

${ }^{7}$ W. Hierse and E. B. Stechel, Phys. Rev. B 50, 17811 (1994).

${ }^{8}$ W. Kohn, Phys. Rev. Lett. 76, 3168 (1996).
${ }^{9}$ E. Hernández and M. J. Gillan, Phys. Rev. B 51, 10157 (1994).

${ }^{10}$ S. Goedecker and L. Colombo, Phys. Rev. Lett. 73, 122 (1994).

${ }^{11}$ A. F. Voter, J. D. Kress, and R. N. Silver, Phys. Rev. B 53, 12 733 (1996).

${ }^{12}$ A. P. Horsfield et al., Phys. Rev. B 53, 2694 (1996).

${ }^{13}$ J. J. P. Stewart, Int. J. Quantum Chem. 58, 133 (1996).

${ }^{14}$ S. Barnett, Matrices: Methods and Applications (Oxford, New York, 1990).

${ }^{15}$ J. P. Perdew, R. G. Parr, M. Levy, and J. L. Balduz, Jr., Phys. Rev. Lett. 49, 1691 (1982).

${ }^{16}$ R. G. Parr and W. Yang, Density-Functional Theory of Atoms and Molecules (Oxford University Press, New York, 1989). 